\title{
Neurological manifestations of primary Sjögren's syndrome
}

\author{
Joanna Perzyńska-Mazan ${ }^{1,2}$, Maria Maślińska3 ${ }^{3}$ Robert Gasik ${ }^{1}$ \\ ${ }^{1}$ Neuroorthopedics and Neurology Clinic and Polyclinic, National Institute of Geriatrics, Rheumatology and Rehabilitation, Warsaw, Poland \\ 2EMG Laboratory, National Institute of Geriatrics, Rheumatology and Rehabilitation, Warsaw, Poland \\ ${ }^{3}$ Clinic of Early Arthritis, National Institute of Geriatrics, Rheumatology and Rehabilitation, Warsaw, Poland
}

\begin{abstract}
Primary Sjögren's syndrome (pSS) is an autoimmune connective tissue disease affecting the exocrine glands, leading to damage of their structure and impairment of their function. In the course of pSS the internal organs may be involved and the symptoms may concern any system. Neurological disorders are one of the most common extraglandular manifestations of pSS. Available literature data estimate the prevalence of neurological symptoms as about $8.5-70 \%$ of patients diagnosed with pSS. The most common neurological complication of pSS is peripheral neuropathy, and in particular sensory polyneuropathy. Central nervous system involvement is much less common. There are also reports of various symptoms connected with damage to cranial nerves and the autonomic nervous system. A careful neurological evaluation, combined with neurophysiological tests, is recommended in patients with pSS. This review summarizes the neurological manifestations of pSS, their possible pathogenic mechanisms, diagnostic evaluation and potential treatment.
\end{abstract}

Key words: primary Sjögren's syndrome, neurological involvement, peripheral neuropathy, neurography.

\section{Introduction}

Primary Sjögren's syndrome (pSS) is an autoimmune connective tissue disease affecting the exocrine glands, leading to damage of their structure and impairment of their function. In the course of pSS the internal organs may be involved and the symptoms may concern any system. Neurological disorders are one of the extraglandular manifestations of the disease and sometimes are very painful. The first reports regarding involvement of the nervous system in the course of pSS were published in 1980 [1]. Literature data estimate the presence of neurological symptoms in about $8.5-70 \%$ of patients diagnosed with pSS [2]. Such a large range results from the fact of applying diverse diagnostic criteria of pSS, different definitions of neurological syndromes as well as greater availability of neurophysiological diagnostics in patients hospitalised at neurology wards as compared with rheumatology wards. In 25-60\% of cases the neurological symptoms preceded the diagnosis of pSS by 2 years on average. In the remaining patients neurological disorders appeared 6-8 years after being diagnosed [2]. The pathogenetic mechanism leading to damage to the nervous system in pSS is not fully known. It is suspected that $T$ lymphocytes and dendritic cells play an important role as they secrete cytokines leading to vasculitis and damage to the dorsal root ganglia as a result of inflammatory infiltration. Specific antibodies reacting with antigens of the nervous tissue are also searched for [3].

The most common neurological complication of pSS is peripheral neuropathy, and in particular sensory polyneuropathy [4]. Central nervous system (CNS) involvement is much less common (2-25\% patients) [5, 6]. In some patients simultaneous involvement of the peripheral and CNS is observed. There are also reports of various symptoms connected with damage to cranial nerves and the autonomic nervous system $[7,8]$.

\section{Neurological manifestations of primary Sjögren's syndrome in the central nervous system}

In the last decade CNS involvement has been observed more commonly than initially suspected. How-

Address for correspondence:

Joanna Perzyńska-Mazan, Neuroorthopedics and Neurology Clinic and Polyclinic, National Institute of Geriatrics, Rheumatology and Rehabilitation, 1 Spartańska St, 02-637 Warsaw, Poland, e-mail: mazanj@wp.pl

Submitted: 05.03.2018; Accepted: 30.03.2018 
ever, as compared with detailed classifications reporting damage to the peripheral nervous system, the knowledge regarding CNS involvement in the case of pSS is not yet as structured as it should be. The following were observed: cognitive disorders, aseptic meningitis, epileptic seizures, headache, transverse myelitis, optic neuritis, disseminated encephalopathy and lesions in the CNS typical of multiple sclerosis.

\section{Cognitive disorders}

Cognitive disorders are common in the case of pSS. The most common forms include attention and memory deficit disorders. Neuropsychological examination shows executive and visuospatial disorders as well as short-term or long-term memory deficits. Magnetic resonance imaging (MRI) of the brain is normal in $80 \%$ of cases or it shows subcortical foci in the fronto-parietal region. Single-photon emission computed tomography (SPECT) may show hypoperfusion areas in the frontal and temporal lobes $[7,9,10]$.

\section{Meningitis}

Meningitis is a relatively common complication of pSS, related to inflammation of the meningeal vessels. The symptoms include headache, flu-like symptoms, confusion, and meningeal signs with or without fever. There may occur focal neurological symptoms in the form of cranial nerve palsy, cerebellum symptoms or seizures. Examination of the cerebrospinal fluid shows aseptic lymphocytic pleocytosis up to 900 cells/ $\mu$ l [2].

\section{Multiple sclerosis-like manifestations}

In $10-20 \%$ of patients diagnosed with pSS there are lesions in the central nervous system analogous to those presented in the case of multiple sclerosis (multiple sclerosis-like lesions). Most often they concern the white matter of the brain (60\%) and the spinal cord $(40 \%)[7,11]$. The observed neurological symptoms include paresis of limbs, aphasia, ataxia, and internuclear ophthalmoplegia; their course is chronic, relapsing-remitting, similar to multiple sclerosis (MS). The cerebrospinal fluid of such patients is characterised by an increased IgG index and synthesis of oligoclonal bands, which is typical of MS [12]. Most patients with multiple sclerosis-like symptoms are also diagnosed with improper visual evoked potentials (VEPs). The symptoms of dryness characteristic of pSS are slightly intensified $[2,13]$.

The reports of pSS also include cases of optic neuritis with transverse myelitis and presence of antibodies against aquaporin-4, imitating Devic's disease, which is a variant of MS [14].

\section{Optic neuritis}

Bilateral retrobulbar optic neuritis has been extensively described among patients diagnosed with pSS. In some cases the first symptom of pSS was blindness secondary to bilateral optic neuritis. In about $12-15 \%$ of cases the features of optic neuritis were found during VEP tests. It is believed that the pathogenesis of optic neuritis in pSS is a combination of demyelination and ischemic vasculitis [7].

\section{Spinal cord involvement}

Acute transverse myelitis is the most common form of spinal cord involvement in the course of pSS. The spectrum of clinical symptoms is diverse, depending on the region of the focal lesion: tetraparesis or paraparesis, sphincter dysfunctions, proprioceptive dysfunction, Brown-Séquard syndrome. The MRI of those patients found hyperintense lesions in T2, present mainly in the cervical region [15]. There are also reports of the form including involvement of the lower motor neuron imitating amyotrophic lateral sclerosis (ALS) [16] and the form including involvement of the upper motor neuron [17].

\section{Neurological manifestations of primary Sjögren's syndrome in the peripheral nervous system}

The main manifestations of involvement of the peripheral nervous system in pSS include various types of neuropathies [6, 18-22]. The presence of peripheral neuropathy in pSS not only decreases the quality of life of a patient, but it is also a negative prognostic factor - it is related to a risk of developing lymphoma [23, 24].

Additionally, there have been reports demonstrating the relation of pSS with motor neuron disease and myositis. It was also observed that patients with pSS reported isolated cases of channelopathy that exhibits features of hypokalaemic paralysis [25].

\section{Peripheral neuropathy}

According to the available data, the frequency of neuropathy in a population of patients with pSS ranges from 2 to $60 \%[18,20,21,26-28]$. The main cause of such a discrepancy may be the application of diverse methods for detection of neuropathy. Some studies were based only on clinical diagnosis, while other researchers applied more or less detailed electrophysiological diagnostics. The reported neuropathies in pSS included distal sensory polyneuropathy, axonal sensorimotor polyneuropathy, chronic inflammatory demyelinating polyneuropathy (CIDP), multiple mononeuropa- 
thy, sensory neuronopathy and small fibre neuropathy. Sensorimotor polyradiculoneuropathy corresponding to Guillain-Barré syndrome was also described [29]. Additionally, the course of pSS may lead to damage of the cranial nerves. Individual types of neuropathies may overlap in one patient $[6,18]$. The variability of neuropathies in patients with pSS and their diverse response to the applied treatment indicates a complex pathomechanism responsible for their occurrence. It is probably different in individual types of neuropathies. One of the main mechanisms for development of neuropathies in pSS is inflammation of the vessel supplying blood to the nerves, in some cases of necrotic nature, with perivascular inflammatory infiltration [30]. An exception is sensory ganglionopathy related mainly to lymphocytic infiltration around the dorsal root ganglia [31]. The role of antibodies in development of neuropathy has not been proven [32].

\section{Distal axonal sensory polyneuropathy}

Distal axonal sensory polyneuropathy, the most common form of neuropathy in pSS, is characterised by a chronic course with a slow increase of symptoms [33]. The symptoms include symmetric paraesthesias and sensory disorders in distal parts of lower limbs. Paraesthesias may be accompanied by burning feet pain. The symptoms are definitely more intense in lower limbs. The upper limbs are involved only in about 20\% of cases. Physical examination shows loss of sensation in a "stocking-glove" pattern and diminished or absent deep tendon reflexes. Electrodiagnostic study demonstrates a symmetric axonal pattern of sensory fibre involvement. Nerve biopsy exhibits axonal degeneration with features of remyelination, without features of necrotic vascular inflammation [2].

\section{Sensorimotor polyneuropathy}

Some patients with distal axonal sensory polyneuropathy also complain of symptoms related to the motor nerve fibres. As the disease progresses, weakening of distal muscles of the limbs, initially of the foot extensors, is observed. Electrophysiological examination reveals axonal sensory and motor loss with significant predominance of the sensory fibres and lower limbs being more affected [7].

\section{Chronic inflammatory demyelinating polyneuropathy}

Chronic inflammatory demyelinating polyneuropathy (CIDP) is uncommon in pSS. It is characterised by slow development (within at least 8 weeks), usually symmetric weakening of proximal and/or distal muscles of upper and lower limbs with sensory dysfunctions and diminished or absent deep tendon reflexes. A characteristic feature of CIDP is increased concentration of protein in the cerebrospinal fluid in $80-90 \%$ of patients. Electrodiagnostic findings include prolonged distal motor latency, slowed conduction velocity, abnormal temporal dispersion or partial conduction block, and absent F-wave or prolongation of its latency [34]. Similarly to the general population, CIDP in patients with pSS is usually treated with intravenous immunoglobulin infusions, plasmapheresis or glucocorticoids (GK) [35].

Cases of acute onset demyelinating polyneuropathy have also been described [29].

\section{Multiple mononeuropathy}

Multiple mononeuropathy means simultaneous or consecutive asymmetric damage of at least two nerves which do not form a continuity with each other. The patients present symptoms in the areas supplied by the affected nerves. Overlapping multifocal lesions of individual nerves may lead to paresis and abnormal sensations imitating polyneuropathy. The researchers do not agree about the frequency of occurrence of multiple mononeuropathy in the course of pSS. According to Mori et al. [8] and Gemignani et al. [20] it occurs in about $12 \%$ of patients with pSS. Terrier et al. [36] and Ramos-Casals et al. [37] indicate that the frequency of neuropathy may be even $50 \%$ of patients with pSS. The main cause of the discussed type of neuropathy is inflammation of the vessels nourishing the nerve trunks with a consequential nerve infarction [38].

Vasculitis is usually not limited to nerves, but also involves other organs, and therefore mononeuropathy in pSS is usually accompanied by acute or subacute constitutional symptoms. Laboratory findings include an elevated erythrocyte sedimentation rate (ESR) and C-reactive protein levels (CRP) and cryoglobulinaemia. Electrophysiological studies document axonal damage and "pseudoblocks" corresponding to the areas of nerve ischaemia. Histopathologically, in those areas, it is possible to visualise necrosis of the vasa nervorum wall of the nerve with concomitant $T$ cell and macrophage infiltration [39].

Clinically, sensory and motor deficits are observed in the distribution of an area innervated by individual nerves. It typically involves acute or subacute onset of the ailment (days, weeks) with accompanying pain, deeply located in the proximal part of the affected limb and painful paraesthesias in the scope of sensory nerve supply. The onset may also be painless with dominating weakness of the limb. Early detected multiple mononeuropathy and proper diagnosis of the underlying vascular inflammation determines successful treatment by means of immuno- 
suppression in the broad sense, usually including GK and immunosuppressive agents (azathioprine, cyclophosphamide, methotrexate, or mycophenolate mofetil) [36].

\section{Sensory ganglionopathy (sensory neuronopathy)}

It regards selective damage to the dorsal root ganglia. All sensory modalities may be distorted, but clinical manifestations are usually dominated by symptoms of damage to the type 1a large sensory fibres, conducting signals from muscle spindles. The main sign is unstable gait with a wide base of support. Unsteadiness of gait results in the fact that sensory neuropathy is a cause of significant immobility of the patients, which often forces them to use a wheelchair. Apart from gait instability, characteristic features include intensified dysfunction of vibration sensation and absent deep tendon reflexes (areflexia) while maintaining proper muscle strength [40, 41].

In some cases involuntary, irregular athetosis-like movements (pseudoathetoid movements) of limbs were observed, suggesting extrapyramidal symptoms. The course of the disease is usually subacute with symptoms aggravating within a few weeks [42]. Sensory neuropathy is usually symmetric, but there are cases where symptoms intensify unilaterally. In contrast to multiple mononeuropathy or distal sensory neuropathy, the symptoms occur along the entire limb as well as in the area of the trunk [40]. Electrodiagnostic study shows reduced or absent sensory nerve action potentials. Somatosensory evoked potential abnormalities are also observed. Motor conduction studies and needle electromyography results remain normal $[3,8,38,40]$. Biopsy shows T lymphocyte infiltration in the dorsal root ganglia and reduction of large fibres. Although sensory ganglionopathy is a common symptom of pSS, no immunological profile characteristic of this type of neuropathy has been found. One recent study indicated that patients with neuropathy are usually seronegative, while the biopsy of the minor salivary glands shows intensive lymphocytic infiltration $(2,28)$. Currently, there are no treatment regimens for sensory neuropathy. So far there have been some trials with immunoglobulins [43, 44], interferon- $\alpha$ [45], D-penicillamine, and rituximab (monoclonal antibody anti-CD20). However, the results of treatment were unsatisfactory.

\section{Small fibre neuropathy}

Small fibre neuropathy occurs from damage to the A- $\delta$ small myelinated fibres and/or unmyelinated C fibres which conduct nociceptive stimuli and temperatures. It is one of the most common types of neuropathy in pSS. It is characterised by very painful, burning paraesthesias (the severity of pain according to the Visual Analogue
Scale being at least 5). The onset is subacute or chronic, lasting for weeks or months. Neurological examination often shows no abnormalities or demonstrates pain sensation dysfunction (decreased pinprick) as well as decreased thermal sensation, with maintained vibratory sensation and proprioception. Contrary to diabetic neuropathy where the symptoms are more intense distally, the neuropathy in pSS concerns more patients who demonstrate symptoms in a non-length-dependent fashion, involving proximal parts of limbs, trunk or face. Conventional sensory nerve conduction studies show no abnormalities. The above fact in combination with the normal result of neurological examination is a cause of frequent suspicion of psychosomatic disorders [46].

In order to confirm the diagnosis of this type of neuropathy, a skin biopsy is performed, which reveals reduction in intra-epidermal nerve fibre density (IENFD). Due to lack of diagnostic tools, the frequency of occurrence of this neuropathy has still not been evaluated. It is assumed that it occurs in $5-10 \%$ of patients with pSS. In some of them the disease also affects larger sensory fibres which conduct the touch sensation or even limb position [47]. Treatment is mainly symptomatic, including tricyclic antidepressants, duloxetine and antiepileptic drugs (gabapentin, duloxetine), the next option being opioids.

\section{Cranial nerve neuropathies}

Sensory trigeminal nerve $(V)$ involvement is the most commonly described type of cranial neuropathy in pSS. It is usually unilateral and affects the middle branch (the maxillary nerve). Patients with pSS have also been diagnosed with facial nerve neuropathy as well as impairment of the cochlear (VIII) nerve, and also with both hearing loss and vestibular symptoms [7]. Mori et al. [8] described multifocal involvement of the $3^{\text {rd }}, 6^{\text {th }}, 5^{\text {th }}, 7^{\text {th }}$, $9^{\text {th }}, 10^{\text {th }}$, and $12^{\text {th }}$ nerves in diverse combinations.

\section{Anterior horn cell damage}

Motor neuron diseases, also called diseases affecting the anterior horn cells, occur in exceptional cases in patients with pSS $[16,48,49]$. In most of the described cases the paresis, atrophies and fasciculations (fast contractions of muscle fibres occurring spontaneously and irregularly) were visible mainly in distal parts of limbs. No accompanying signs of damage to the upper motor neuron were found. Electrodiagnostic study showed normal sensory nerve conduction. Motor nerve conduction studies demonstrated reduced (compound muscle action potential) CMAP amplitudes and prolonged latency or absence of the F-wave. The needle examination showed signs of acute denervation (fibrillation potentials, positive sharp waves) and fasciculation potentials (spontaneous 
involuntary discharge of single motor units). Histopathological examination demonstrated mononuclear cell infiltration in the area of motor neurons and axon degeneration. Atrophy and motor root fibrosis were also observed (a result corresponding to prolonged F-wave latency or absent F-wave). No features of vascular inflammation were found. Histopathological examination of the upper motor neurons was normal [50]. Improvement after treatment with corticosteroids and other immunosuppressive drugs was reported [51].

\section{Myositis in primary Sjögren's syndrome}

Mild myositis was documented in some patients with pSS, reporting fatigability and myalgia-like symptoms. Discrete histopathological lesions, suggesting myositis, may be found in most patients. However, clinical symptoms of myositis with relevant laboratory, electrophysiological and histopathological test results regard a few per cent of patients with pSS [52]. Some reports have included presence of histopathological lesions similar to those presented in the case of inclusion body myositis [53, 54].

\section{Hypokalaemic periodic paralysis in primary Sjögren's syndrome}

So far only single cases of hypokalaemic periodic paralysis in pSS have been described with complications of renal tubular acidosis (RTA) $[25,55]$. One patient was also diagnosed with vitamin D deficiency. The symptoms of dryness were mild or absent, despite the positive result of the Schirmer test and positive result of the minor salivary gland biopsy. Supplementation with potassium, vitamin D and glucocorticosteroids allowed remission of the symptoms to be achieved.

\section{Neurological manifestations of primary Sjögren's syndrome in the autonomic nervous system}

Disorders of the autonomic nervous system (dysautonomia) in pSS were diagnosed with variable frequency from 3 to $50 \%[8,56]$. The most common symptoms included orthostatic hypotension, heart arrhythmia, disorders of gastrointestinal motor activity, bladder dysfunction, secretomotor dysfunction, and Adie's syndrome. The actual frequency of dysautonomia is difficult to evaluate due to diverse definitions and use of various methods for assessment of the autonomic system. Additionally, the function of the autonomic system is modulated by many factors, including emotional conditions, time of the day, temperature of the environment, stimulants, and drugs.
Involvement of the autonomic systems in pSS is caused by various mechanisms, different for different organs. Most symptoms are connected with type 3 muscarinic receptor blocking. The cause of development of dysautonomia may include cytokines interfering with cholinergic neurotransmission, destruction of autonomic nerve fibres of the external secretion gland cells, and inflammatory $T$ lymphocytic infiltration in the area of root ganglia and nerves of the autonomic nervous system [57-59]. Additionally, patients with pSS have an increased level of cholinesterase (an enzyme which hydrolyses Ach) [60].

The function of the autonomic nervous system is most often examined using the orthostatic test and quantitative sudomotor axon reflex test (QSART). Apart from clinical assessment, the following electrophysiological methods are used: microneurography, sympathetic skin response and measurement of the heart rate variability (HRV). The first two tests evaluate the function of the sympathetic system. HRV analysis evaluating the cardiovascular autonomic nervous system (ANS) function does not always reflect the autonomic nervous function in other organs.

\section{Conclusions}

Involvement of the nervous system in pSS is a negative prognostic factor. It is usually related to a more aggressive course of the disease. Patients diagnosed with pSS should be regularly examined by means of the ESSDAI (EULAR Sjögren syndrome disease activity index). The index includes domains which take into account damage to the muscles, peripheral nerves and central nervous system. In the case of damage to the nervous system more intensive treatment should be indicated. The need for precise neurological diagnostics should be stressed in the case of patients with pSS, and even in the case of a patient without any symptoms or signs of damage to the nervous system. Considering neurophysiological tests may contribute to detection of early stage of the damage to the nervous system, even in the pre-clinical phase.

The authors declare no conflict of interest.

\section{References}

1. Alexander E, Provost T, Stevens M, et al. Neurologic complications of primary Sjögren's syndrome. Medicine (Baltimore) 1982; 61: 247-257.

2. Fauchais AL, Magy L, Vidal E. Central and peripheral neurological complications of primary Sjögren's syndrome. Presse Med 2012; 41: 485-493. 
3. Griffin JW, Cornblath DR, Alexander E, et al. Ataxic sensory neuropathy and dorsal root ganglionitis associated with Sjögren's syndrome. Ann Neurol 1990; 27: 304-315.

4. Chai J, Logigian EL. Neurological manifestations of primary Sjögren syndrome. Curr Opin Neurol 2010; 23: 509-513.

5. Moutsopoulos HN, Sarmas JH, Talal N. Is central nervous system involvement a systemic manifestation of primary Sjögren syndrome? Rheum Dis Clin North Am 1993; 19: 909-912.

6. Andonopoulos AP, Lagos G, Drosos AA, et al. The spectrum of neurological involvement in Sjögren syndrome. Br J Rheumatol 1990; 29: 21-23.

7. Delalande S, de Seze J, Fauchais AL, et al. Neurologic manifestations in primary Sjögren syndrome: a study of 82 patients. Medicine (Baltimore) 2004; 83: 280e91.

8. Mori K, lijima $M$, Koike $H$, et al. The wide spectrum of clinical manifestations in Sjögren's syndrome-associated neuropathy. Brain 2005; 128: 2518-2534.

9. Le Guern V, Belin C, Henegar C, et al. Cognitive function and 99mTc-ECD brain SPECT are significantly correlated in patients with primary Sjögren syndrome: a case-control study. Ann Rheum Dis 2010; 69: 132-137.

10. Mataró M, Escudero D, Ariza M, et al. Magnetic resonance abnormalities associated with cognitive dysfunction in primary Sjögren syndrome. J Neurol 2003; 250: 1070-1076.

11. Alexander EL, Malinow K, Lejewski JE, et al. Primary Sjögren's syndrome with central nervous system disease mimicking multiple sclerosis. Ann Intern Med 1986; 104: 323-330.

12. Thong BY, Venketasubramanian N. A case of Sjögren's syndrome or multiple sclerosis? A diagnostic and therapeutic dilemma. Rheumatol Int 2002; 22: 256-258.

13. Pillemer SR, Mendelsohn AB, Morgen KE. Central Nervous Sys tem involvement. In: Sjögren's Syndrome. Ramos-Casals M (ed.). Springer-Verlag London Ltd, London 2012: 281-291.

14. de Seze J, Devos D, Castelnovo G, et al. The prevalence of Sjögren syndrome in patients with primary progressive multiple sclerosis. Neurology 2001; 57: 1359-1363.

15. de Seze J, Delalande S, Fauchais AL, et al. Myelopathies secondary to Sjögren's syndrome: treatment with monthly intravenous cyclophosphamide associated with corticosteroids. J Rheumatol 2006; 33: 709-711.

16. Zahlane S, Louhab N, El Mellakh M, et al. Anterior horn syndrome: A rare manifestation of primary Sjögren's syndrome. Joint Bone Spine 2016; 83: 448-450.

17. Hagiwara K, Murai $\mathrm{H}$, Ochi $\mathrm{H}$, et al. Upper motor neuron syndrome associated with subclinical Sjögren's syndrome. Intern Med 2008; 47: 1047-1051.

18. Gøransson LG, Herigstad A, Tjensvoll AB, et al. Peripheral neuropathy in primary Sjögren syndrome: a population-based study. Arch Neurol 2006; 63: 1612-1615.

19. Vrethem $M$, Lindvall B, Holmgren $H$, et al. Neuropathy and myopathy in primary Sjögren's syndrome: neurophysiological, immunological and muscle biopsy results. Acta Neurol Scand 1990; 82: 126-131.

20. Gemignani F, Marbini A, Pavesi G, et al. Peripheral neuropathy associated with primary Sjögren's syndrome. J Neurol Neurosurg Psychiatry 1994; 57: 983-986.
21. Barendregt PJ, van den Bent MJ, van Raaij-van den Aarssen VJ, et al. Involvement of the peripheral nervous system in primary Sjögren's syndrome. Ann Rheum Dis 2001; 60: 876-881.

22. Lopate G, Pestronk A, Al-Lozi M, et al. Peripheral neuropathy in an outpatient cohort of patients with Sjögren's syndrome. Muscle Nerve 2006; 33: 672-676.

23. Skopouli FN, Dafni U, Ioannidis JP, et al. Clinical evolution, and morbidity and mortality of primary Sjögren's syndrome. Semin Arthritis Rheum 2000; 29: 296-304.

24. Ioannidis JP, Vassiliou VA, Moutsopoulos HM. Long-term risk of mortality and lymphoproliferative disease and predictive classification of primary Sjögren's syndrome. Arthritis Rheum 2002; 46: 741-747.

25. Liao CY, Wang CC, Chen IH, et al. Hypokalemic paralysis as a presenting manifestation of primary Sjögren's syndrome accompanied by vitamin D deficiency. Intern Med 2013; 52 : 2351-2353.

26. Garcia-Carrasco M, Ramos-Casals M, Rosas J. Primary Sjögren syndrome: Clinical and immunologic disease patterns in a cohort of 400 patients. Medicine (Baltimore) 2002; 81: 270-280.

27. Lafitte C, Amoura Z, Cacoub P, et al. Neurological complications of primary Sjögren's syndrome. J Neurol 2001; 248: 577584.

28. Sène D, Jallouli $M$, Lefaucheur J-P, et al. Peripheral neuropathies associated with primary Sjögren syndrome: immunologic profiles of nonataxic sensory neuropathy and sensorimotor neuropathy. Medicine (Baltimore) 2011; 90: 133-138.

29. Tanaka K, Nakayasu H, Suto Y, et al. Acute Motor-dominant Polyneuropathy as Guillain-Barré Syndrome and Multiple Mononeuropathies in a Patient with Sjögren's Syndrome. Intern Med 2016; 55: 2717-2722.

30. Tobón GJ, Pers JO, Devauchelle-Pensec V, et al. Neurological Disorders in Primary Sjögren's Syndrome. Autoimmune Dis 2012; 2012: 645967.

31. Camdessanché JP, Jousserand G, Ferraud K, et al. The pattern and diagnostic criteria of sensory neuronopathy: a case-control study. Brain 2009; 132: 1723-1733.

32. Jamilloux Y, Magy L, Hurtevent JF, et al. Immunological profiles determine neurological involvement in Sjögren's syndrome. Eur J Intern Med 2014; 25: 177-181.

33. Mochizuki H, Kamakura K, Masaki T, et al. Motor dominant neuropathy in Sjögren's syndrome: report of two cases. Intern Med 2002; 41: 142-146.

34. Dworkin RH, O'Connor AB, Backonja M, et al. Pharmacologic management of neuropathic pain: evidence-based recommendations. Pain 2007; 132: 237-251.

35. Hughes RA, Donofrio P, Bril V, et al. Intravenous immune globulin (10\% caprylate-chromatography purified) for the treatment of chronic inflammatory demyelinating polyradiculoneuropathy (ICE study): a randomised placebo-controlled trial. Lancet Neurol 2008; 7: 136-144.

36. Terrier B, Lacroix C, Guillevin L, et al. Diagnostic and prognostic relevance of neuromuscular biopsy in primary Sjögren's syndrome-related neuropathy. Arthritis Rheum 2007; 57: 1520-1529.

37. Ramos-Casals M, Solans R, Rosas J, et al. Primary Sjögren's syndrome in Spain: clinical and immunologic expression in 1010 patients. Medicine (Baltimore) 2008; 87: 210-219. 
38. Grant IA, Hunder GG, Homburger HA, et al. Peripheral neuropathy associated with sicca complex. Neurology 1997; 48 855-862.

39. England JD, Gronseth GS, Franklin G, et al. Evaluation of distal symmetric polyneuropathy: the role of autonomic testing, nerve biopsy, and skin biopsy (an evidence-based review). Muscle Nerve 2009; 39: 106-115.

40. Dalakas MC. Chronic idiopathic ataxic neuropathy. Ann Neuro 1986; 19: 545-554.

41. Pereira PR, Viala K, Maisonobe T, et al. Sjögren Sensory Neuronopathy (Sjögren Ganglionopathy): Long-Term Outcome and Treatment Response in a Series of 13 Cases. Medicine (Baltimore) 2016; 95: e3632.

42. Souayah N, Chong PS, Cros D. Acute sensory neuronopathy as the presenting symptom of Sjögren's syndrome. J Clin Neurosci 2006; 13: 862-865.

43. Takahashi Y, Takata T, Hoshino M, et al. Benefit of IVIG for long-standing ataxic sensory neuronopathy with Sjögren's syndrome. IV immunoglobulin. Neurology 2003; 60: 503-505.

44. Chen WH, Yeh JH, Chiu HC. Plasmapheresis in the treatment of ataxic sensory neuropathy associated with Sjögren's syndrome. Eur Neurol 2001; 45: 270-274.

45. Yamada S, Mori K, Matsuo K, et al. Interferon alfa treatment for Sjögren's syndrome associated neuropathy. J Neurol Neurosurg Psychiatry 2005; 76: 576-578.

46. Lacomis D. Small-fiber neuropathy. Muscle Nerve 2002; 26: 173-188.

47. Mori K, lijima M, Sugiura M, et al. Sjögren's syndrome associated painful sensory neuropathy without sensory ataxia. J Neurol Neurosurg Psychiatry 2003; 74: 1320-1322.

48. Amoura Z, Salachas F, Cacoub B, et al. Pseudosclerose laterale amyotrophiqueau tours du syndrome de Gougerot-Sjögren primitif. Rev Med Interne 1999; 20: 83s.

49. Attout L, Rahmeh F, Ziegler L. Syndrome de Gougerot-Sjögren simulant unesclerose laterale amyotrophique. Rev Med Interne 2000; 21: 708-710.

50. Katz JS, Houroupian D, Ross MA. Multisystem neuronal involvement and sicca complex: broadening the spectrum of complications. Muscle Nerve 1999; 22: 404-407.

51. Rafai MA, Boulaajaj FZ, Moutawakil F, et al. Neurological manifestations revealing primitive Gougerot-Sjögren syndrome: 9 cases. Joint Bone Spine 2009; 76: 139-145.

52. Lindvall B, Bengtsson A, Ernerudh J, et al. Subclinical myositis is common in primary Sjögren's syndrome and is not related to muscle pain. J Rheumatol 2002; 29: 717-725.

53. Kanellopoulos P, Baltoyiannis C, Tzioufas AG. Primary Sjögren's syndrome associated with inclusion body myositis. Rheumatology (Oxford) 2002; 41: 440-444.

54. Misterska-Skóra M, Sebastian A, Dzięgiel P, et al. Inclusion body myositis associated with Sjögren's syndrome. Rheumatol Int 2013; 33: 3083-3086.

55. Hung YM, Huang NC, Wann SR, et al. Recurrent Hypokalemic Periodic Paralysis UnmasksSjögren Syndrome without Sicca Symptoms. J Coll Physicians Surg Pak 2015; 25 Suppl 1 : S36-S38.

56. Andonopoulos AP, Christodoulou J, Ballas C, et al. Autonom ic cardiovascular neuropathy in Sjögren's syndrome. A controlled study. J Rheumatol 1998; 25: 2385-2388.
57. Waterman SA, Gordon TP, Rischmueller M. Inhibitory effects of muscarinic receptor autoantibodies on parasympathetic neurotransmission in Sjögren's syndrome. Arthritis Rheum 2000; 43: 1647-1654.

58. Dawson L, Tobin A, Smith P, et al. Antimuscarinic antibodies in Sjögren's syndrome: where are we, and where are we going? Arthritis Rheum 2005; 52: 2984-2995.

59. Fox RI, Stern M. Sjögren's syndrome: mechanisms of pathogenesis involve interaction of immune and neurosecretory systems. Scand J Rheumatol 2002; 116: 3-13.

60. Dawson LJ, Caulfield VL, Stanbury JB, et al. Hydroxychloroquine therapy in patients with primary Sjögren's syndrome may improve salivary gland hypofunction by inhibition of glandular cholinesterase. Rheumatology 2005; 44: 449-455. 